\title{
Selection signature reveals genes associated with susceptibility loci affecting respiratory disease due to pleiotropic and hitchhiking effect in Chinese indigenous pigs
}

\author{
Zhong Xu' ${ }^{1}$, Hao Sun ${ }^{1}$, Zhe Zhang ${ }^{1}$, Cheng-Yue Zhang ${ }^{1}$, Qing-bo Zhao ${ }^{1}$, Qian Xiao ${ }^{1}$, \\ Babatunde Shittu Olasege ${ }^{1}$, Pei-Pei Ma ${ }^{1}$, Xiang-Zhe Zhang ${ }^{1}$, Qi-Shan Wang ${ }^{1, *}$, and Yu-Chun Pan ${ }^{1,2, *}$
}

\author{
* Corresponding Authors: \\ Qi-Shan Wang \\ Tel: +86-21-34205836, Fax: +86-21-34206394, \\ E-mail: wangqishan@sjtu.edu.cn \\ Yu-Chun Pan \\ Tel: +86-21-34205836, Fax: +86-21-34206394 \\ E-mail: panyuchun1963@aliyun.com
}

${ }^{1}$ Department of Animal Science, School of Agriculture and Biology, Shanghai Jiao Tong University, Shanghai 200240, China

2 Shanghai Key Laboratory of Veterinary Biotechnology, Shanghai 200240, China

ORCID

Zhong Xu

https://orcid.org/0000-0002-9009-0200

Hao Sun

https://orcid.org/0000-0002-0542-9477

Zhe Zhang

https://orcid.org/0000-0001-5320-3125

Cheng-Yue Zhang

https://orcid.org/0000-0002-4148-7201

Qing-bo Zhao

https://orcid.org/0000-0002-5423-1029

Qian Xiao

https://orcid.org/0000-0002-6917-5402

Babatunde Shittu Olasege

https://orcid.org/0000-0001-8954-5768

Pei-Pei Ma

https://orcid.org/0000-0002-2718-0465

Xiang-Zhe Zhang

https://orcid.org/0000-0001-5419-7679

Qi-Shan Wang

https://orcid.org/0000-0002-6475-0009

Yu-Chun Pan

https://orcid.org/0000-0002-1163-5963

Submitted Sept 3, 2018; Revised Nov 20, 2018; Accepted Jan 14, 2019
Objective: Porcine respiratory disease is one of the most important health problems causing significant economic losses. To understand the genetic basis for susceptibility to swine enzootic pneumonia (EP) in pigs, we detected 102,809 single nucleotide polymorphisms in a total of 249 individuals based on genome-wide sequencing data.

Methods: Genome comparison of susceptibility to swine EP in three pig breeds (Jinhua, Erhualian, and Meishan) with two western lines that are considered more resistant (Duroc and Landrace) using cross-population extended haplotype homozygosity and F-statistic $\left(\mathrm{F}_{\mathrm{ST}}\right)$ statistical approaches identified 691 positively selected genes. Based on quantitative trait loci, gene ontology terms and literature search, we selected 14 candidate genes that have convincible biological functions associated with swine $\mathrm{EP}$ or human asthma.

Results: Most of these genes were tested by several methods including transcription analysis and candidate genes association study. Among these genes: cytochrome P450 1A1 and catenin beta 1 (CTNNB1) are involved in fertility; transforming growth factor beta receptor 3 plays a role in meat quality traits; Wnt family member 2, CTNNB1 and transcription factor 7 take part in adipogenesis and fat deposition simultaneously; plasminogen activator, urokinase receptor (completely linked to AXL receptor tyrosine kinase, $\mathrm{r}^{2}=1$ ) plays an essential role in the successful ovulation of matured oocytes in pigs; colipase like 2 (strongly linked to SAM pointed domain containing ETS transcription factor, $\mathrm{r}^{2}=0.848$ ) is involved in male fertility. Conclusion: These adverse genes susceptible to swine EP may be selected while selecting for economic traits (especially reproduction traits) due to pleiotropic and hitchhiking effect of linked genes. Our study provided a completely new point of view to understand the genetic basis for susceptibility or resistance to swine EP in pigs thereby, provides insight for designing sustainable breed selection programs. Finally, the candidate genes are crucial due to their potential roles in respiratory diseases in a large number of species, including human.

Keywords: Pigs; Selection Signature; Respiratory Disease; XP-EHH; $\mathrm{F}_{\mathrm{ST}}$

\section{INTRODUCTION}

Porcine respiratory disease is one of the most important health problems associated with swine production [1]. It can be caused by various infectious pathogens, such as viruses, mycoplasma, bacteria and parasites. Swine enzootic pneumonia (EP) caused by Mycoplasma hyopneumoniae (Mhp) is a chronic and endemic respiratory disease, which has similar pathogenesis and clinical symptoms to human asthma [2]. Mhp infections are highly prevalent in almost all swine producing areas, causing significant economic losses due to decreased growth rate, poor feed efficiency and increased cost of healthcare. Both the control and eradication of EP are difficult because of its complicated mechanisms of infection and its 
co-infection with other respiratory pathogens such as Mycoplasma hyorhinis, Pasteurella multocida, porcine respiratory and reproductive syndrome virus (PRRSV), and porcine circovirus type 2. Many efforts including vaccination, medication and sanitation have been devoted to controlling its prevalence. However, the frequent application of antibiotics can increase the incidence of drug resistant bacteria and also raise the level of antibiotic residue in food.

Recently, the genetic improvement of disease resistance in animals has been attracting attention. Several studies have explored the genetic mechanisms of susceptibility to EP. Okamura et al [3] improved a Landrace breed by selecting for swine EP resistance and meat production over five generations. They identified a significant quantitative trait locus (QTL) for EP between microsatellite markers SW1650 and SW240 on S.scrofa chromosome 2. Huang et al [4] conducted a genome-wide association studies on 332 Chinese Erhualian pigs which were genotyped using Illumina Porcine $60 \mathrm{~K}$ single nucleotide polymorphism (SNP) chips, and found C-XC motif chemokine ligand 6 (CXCL6), CXCL8, KIT protooncogene, receptor tyrosine kinase, and C-terminal binding protein 2 as candidate genes that might play important roles in determining resistance or susceptibility to swine EP-like respiratory disease.

In the previous researches and practical production, we noticed that Chinese indigenous pig breeds such as Meishan pigs, Erhualian pigs, and Jinhua pigs are more sensitive to $M h p$ compared with commercial Western pig breeds in the same rearing environment $[4,5]$. By coincidence, these pig breeds show an excellent fertility and meat quality compared to European breeds and other Chinese breeds under the natural and artificial selective pressure. We proposed two hypotheses: One possibility is that the susceptible loci affecting swine $\mathrm{EP}$ are closely linked to the genes or regulatory elements involved in fertility and meat quality traits, and these genes or regulatory elements may have a hitch-hiking effect during the selection for fertility and meat quality traits; another possibility is that the pleiotropic genes or regulatory elements involved in fertility and meat quality traits might simultaneously play important roles in determining susceptibility to swine EP.

To test these hypotheses, we compared the genomes of three Chinese indigenous pig breeds which are very sensitive to swine EP with two commercial pig breeds using crosspopulation extended haplotype homozygosity (XP-EHH) and F-statistic $\left(\mathrm{F}_{\mathrm{ST}}\right)$ approaches [6,7] to detect genomic regions under positive selection that are related to susceptibility to swine EP. Recently, extensive researches have been carried to identify the positive selection of genes in relation to different specific traits using a distorted pattern of genetic variation between populations. For example, positive selection identified several candidate genes involved in Berkshire meat quality [8]. Taye et al [9] also discovered positively selected genes in African cattle responsible for thermotolerance, and Yuan et al [10] revealed several genes associated with tail type in Chinese indigenous sheep.

The aim of this study is to identify genes associated with susceptibility to respiratory disease like swine EP in Chinese indigenous populations using a genomic scan of selective sweep signatures, and to explore how it happened. Understanding the genetic mechanisms in pigs could help to design sustainable breed selection programs.

\section{MATERIALS AND METHODS}

\section{Animals and genotyping}

A total of 249 individuals were considered in this study. There were three pig breeds sensitive to swine EP consisting of 53 Jinhua pigs, 31 Erhualian pigs, and 80 Meishan pigs (50 Middle Meishan and 30 Small Meishan pigs), and two breeds relatively insensitive to swine EP including 48 Duroc pigs and 37 Landrace pigs. The sequencing data of Jinhua pigs were obtained from Li et al [11], Erhualian and Meishan pigs were retrieved from Wang et al [12], Duroc and Landrace pigs were acquired from Zhang et al [13]. All individuals were genotyped using the genotyping by genome reducing and sequencing (GGRS) protocol [14].

The Sscrofa10.2 pig reference genome was used for calling SNPs via SAMtools (version 0.1.19). Quality control of the fastq file was performed using NGS QC Toolkit v2.3, and the parameters were set according to the report from Chen et al [14]. Clean sequencing reads were subsequently mapped to the pig reference genome (Sscrofa10.2) using BWA v0.7.5 with default settings for single-end mapping. The SNP calling was performed using SAMtools software, and the missing genotypes were imputed using BEAGLE v4.1. Some filters were applied to SNPs as follows: i) minor allele frequency $\leq 0.05$; ii) minimum number of individuals with genotyping $\leq 35 \%$; and iii) SNPs on chromosome X. We filtered the SNPs on chromosome $\mathrm{X}$ in consideration of the extremely ancient interspecies introgression and low rate of recombination.

\section{Population structure analysis}

To assess the relationships between the animals and breeds under investigation, several procedures were carried out. To generate a pruned subset of SNPs that are in approximate linkage equilibrium with each other in multidimensional scaling (MDS) analysis, the "--indep 5052 " command was used in PLINK v1.07 [15]. Principal component analysis (PCA) was performed using GCTA software (version 1.24), which represented the population structure based on genetic correlations between individuals. Pairwise identity-by-state distances between all individuals were calculated using PLINK. 


\section{Genome scans for selection signatures}

Before statistical analysis, Jinhua, Erhualian, and Meishan pigs were clustered into Group1 (observed group), whereas Duroc and Landrace pigs were clustered into Group2 (reference groups). To determine a genome wide pattern of positive selection between Group 1 and Group2, $\mathrm{F}_{\mathrm{ST}}$ values per-SNP were calculated based on the formulae proposed by Weir and Cockerham [7]. All $\mathrm{F}_{\mathrm{ST}}$ values in this study are for a single locus. To determine if Group1 has undergone selection, we computed the XP-EHH values using haplotype information in the xpehh program [6]. The phased haplotype data was reconstructed using FASTPHASE (http://stephenslab.uchicago. edu/software.html). The XP-EHH values were estimated by calculating $\mathrm{EHH}$ and log-ratio iHH by comparing haplotypes between Group1 and Group2. EHH and REHH values were calculated using SWEEP v.1.1 (http://www.broadinstitute. org/mpg/sweep/) software.

\section{Gene annotation}

We searched for positional candidate genes in a 20-kb region centering on the selected SNPs using the NCBI database and Ensembl pig genome databases (http://uswest.ensembl.org/ Sus_scrofa/Info/Index). To further analyze the functions of identified genes, Kyoto encyclopedia of genes and genomes (KEGG) pathway and gene ontology (GO) enrichment analyses were performed using the Database for Annotation, Visualization and Integrated Discovery (DAVID). Due to the sparsity of the porcine gene database, we corresponded these genes with the human genome by aligning human ensemble ID. Only terms with a p-value (Benjamini correction) less than 0.05 were considered as significant and listed.

Selection strategy for candidate genes involved in the susceptibility to swine enzootic pneumonia

To select genes that contribute to the susceptibility to swine EP from the total genes with strong signatures of selection, we used the following selection strategies: i) the pig QTL (SS_10.2) database was downloaded from the animal QTL database website (https://www.animalgenome.org/cgi-bin/ QTLdb/SS/index). Because the number of QTLs of Mycoplasmal pneumonia susceptibility (MPS) trait is still relatively few, selection strategy; ii) used the keyword "Mycoplasma" find the MPS relevant traits, and then the genes overlapped with the QTLs were selected as groupA. Meanwhile, the genes matched to GO terms associated with lung, immune and inflammatory response were selected as groupB; iii) We merged these two groups and then the plausible genes were manually selected based on literature search, GeneCard and their biological function.

Transcription analysis based on gene expression omnibus To complement and verify the selected candidate genes, tran- scription analysis based on gene expression omnibus (GEO) was conducted. The only one published Expression profiling microarray (GSE49882, GPL17577) of the porcine alveolar macrophage infected by porcine $M h p$ was downloaded from public GEO database [16], and differentially expressed genes were identified by GEO2R using default parameters. A threshold was applied based on a fold change (FC) of $2.0(\mathrm{p} \leq 0.05)$ between infected $(6 \mathrm{~h} M h p)$ and control (6hControl) cells.

\section{Candidate genes association study}

In this analysis, all 171 Jinhua pigs were treated similarly. The individuals were genotyped using the same genotyping by GGRS protocol. The phenotypes were recorded based on Huang et al [4]. Briefly, one EP score was recorded for an individual which was found with a dry cough or obvious abdominal fluctuations and suffering from fast breathing and loss of appetite per day during 100 to 200 days of age. According to their EP scores, these pigs were grouped into controls $(\mathrm{EP}$ score $=0)$ and cases $(\mathrm{EP}$ score $\geq 1)$. Then we calculated the allele frequencies in the candidate genes between case and control groups and the p-values of SNPs were calculated by Fisher's exact test.

\section{Calculation of $\mathbf{r}^{2}$}

In order to search for potential genes linked to these candidate genes involved in the susceptibility to swine EP owing to the hitchhiking effect or lack of recombination, we calculated the linkage disequilibrium (LD) scores between SNPs located in these candidate genes and the other SNPs in selected regions. The $\mathrm{r}^{2}$ between two SNPs was then computed using PLINK v1.07 [15]. In this study, two genes were considered to be connected if the SNPs located in these two genes were physically close to each other and were subject to strong $\mathrm{LD}$ (usually measured by $\mathrm{r}^{2} \geq 0.8$ ).

\section{RESULTS AND DISCUSSION}

\section{Single nucleotide polymorphism quality control}

After quality control, there were 249 subjects and 102,809 SNPs in the analyzed dataset. The variants were distributed on each chromosome in a relatively uniform fashion, with the exception of some isolated regions on some chromosomes (Figure 1). The SNPs positions within the chromosomes were based on the pig reference genome (SGSC Sscrofa10.2).

\section{Population structure}

To assess the relationships between the animals and breeds under investigation, we applied MDS to analyze 249 individuals using the pruned 35,104 SNPs with low LD extents. The PCA results showed that the first two principal components (PC1 and PC2) explained $19.8 \%$ and $6.5 \%$ of the variance respectively. The PC1 separated all individuals into two non- 


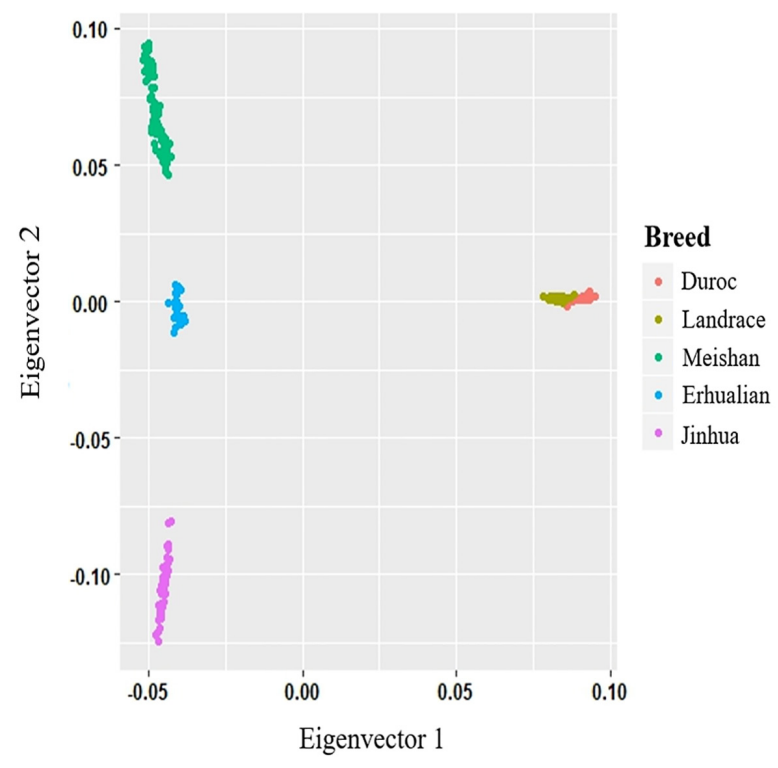

Figure 1. The distribution of the SNPS across the chromosomes. The $x$-axis denotes the chromosomal position (Mb), and the $y$-axis represents the chromosomes. The number of the SNPs present in each 1,000 kb genome block is expressed via colours. SNPs, single nucleotide polymorphisms.

overlapping clusters (Figure 2). Our clustering results showed that the Jinhua, Erhualian, and Meishan pigs were grouped together as one group and that the Duroc, Landrace pigs belonged to another group.

\section{Positive selection signature of swine enzootic pneumonia sensitive populations}

In this study, selection signature analysis was conducted using $\mathrm{XP}-\mathrm{EHH}$ and $\mathrm{F}_{\mathrm{ST}}$ approaches. We used an empirical procedure simultaneously with significantly high XP-EHH (10\% right tail, where $\mathrm{XP}-\mathrm{EHH}$ is 0.50 ) and $\mathrm{F}_{\mathrm{ST}}$ values (10\% right tail, where $\mathrm{F}_{\mathrm{ST}}$ is 0.54 ) of the empirical distribution to clarify regions with strong selective sweep signals along the genome, which should harbor genes that underwent a selective sweep. Consequently, we identified a subset of 1,479 SNPs with strong selective sweep signals (Figure 3A), which exhibited significant differences $\left(\mathrm{p}<2.2 \times 10^{-16}\right.$, MannWhitney U test) in XP-EHH and $\mathrm{F}_{\mathrm{ST}}$ values when compared to whole genomic background (Figure 3B). Finally, these SNPs represented 691 genes with strong signatures of selection (Supplementary Table S1), and only two genes, RNA polymerase III subunit $\mathrm{H}$ and aconitase 2 (ACO2), were embedded in the most significantly selected regions simultaneously with XP-EHH values ( $1 \%$ right tail) and $\mathrm{F}_{\mathrm{ST}}$ values (1\% right tail) (Figure 3C). ACO2 was involved in tricarboxylic acid (TCA) cycle, and hypoxia, a fundamental characteristic of respiratory disease, can regulate the gene expression of mitochondrial aconitase [17].

Using the default settings within DAVID, GO terms, and KEGG pathways with p-value $<0.05$ were enriched and shown in Table 1 . The terms overrepresented were related to cell adhesion, membrane part, membrane. In the KEGG analysis, 10 significant pathways were also identified: such as retinol metabolism, pentose and glucuronate interconversions, metabolism of xenobiotics by cytochrome P450, androgen and estrogen metabolism, starch and sucrose metabolism and porphyrin and chlorophyll metabolism.

Positive selection signature related to susceptibility to swine enzootic pneumonia in Chinese indigenous pigs While selective sweep signals are likely to be detected among various regions, in this paper, we focused on and discussed the genes and pathways that putatively contribute to the swine

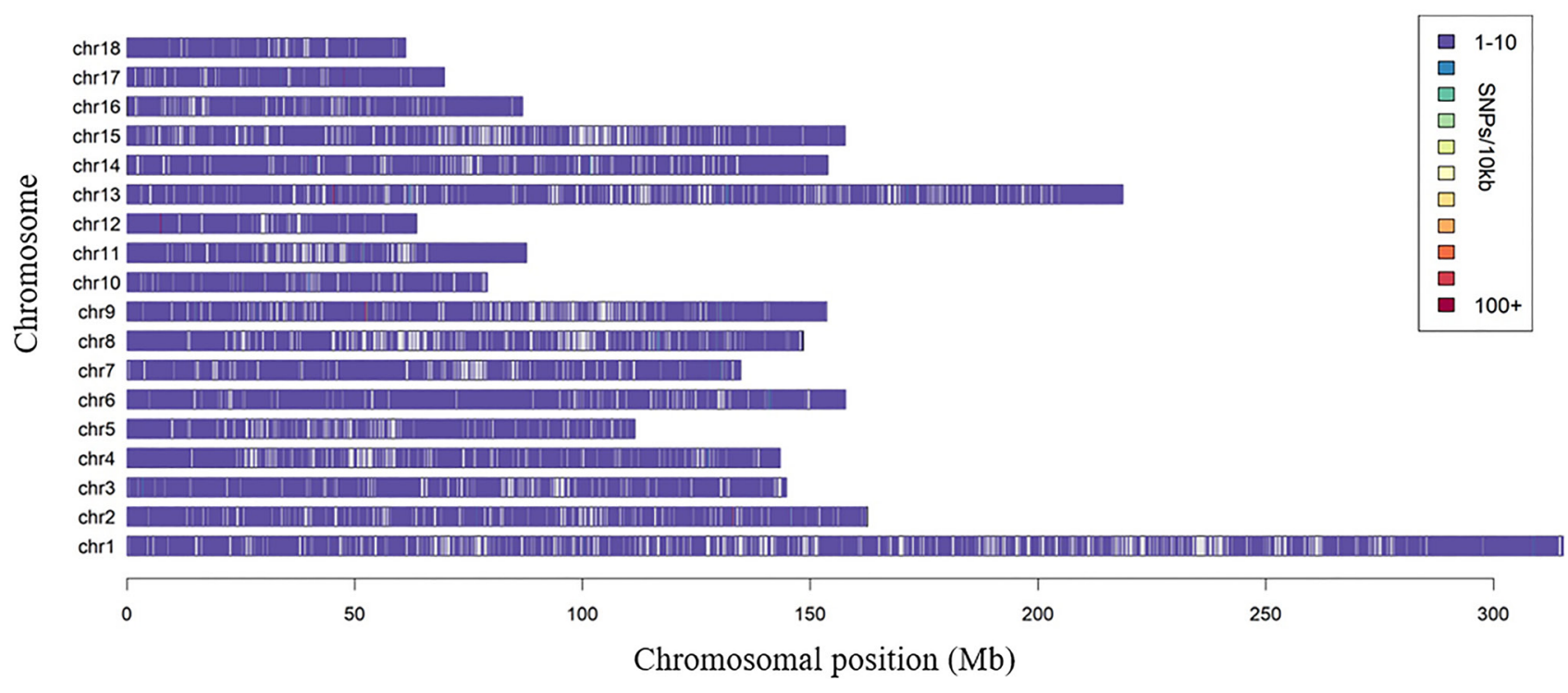

Figure 2. Population structure analysis of all individuals. Results of principal component analysis (PCA) of Duroc, Landrace, Jinhua, Erhualian, and Meishan breeds. Eigenvector1 ( $\mathrm{x}$-axis) versus Eigenvector2 (y-axis). The first two principal components, $\mathrm{PC1}$ and PC2, account for $19.8 \%$ and $6.5 \%$ of the total variance, respectively. 
a

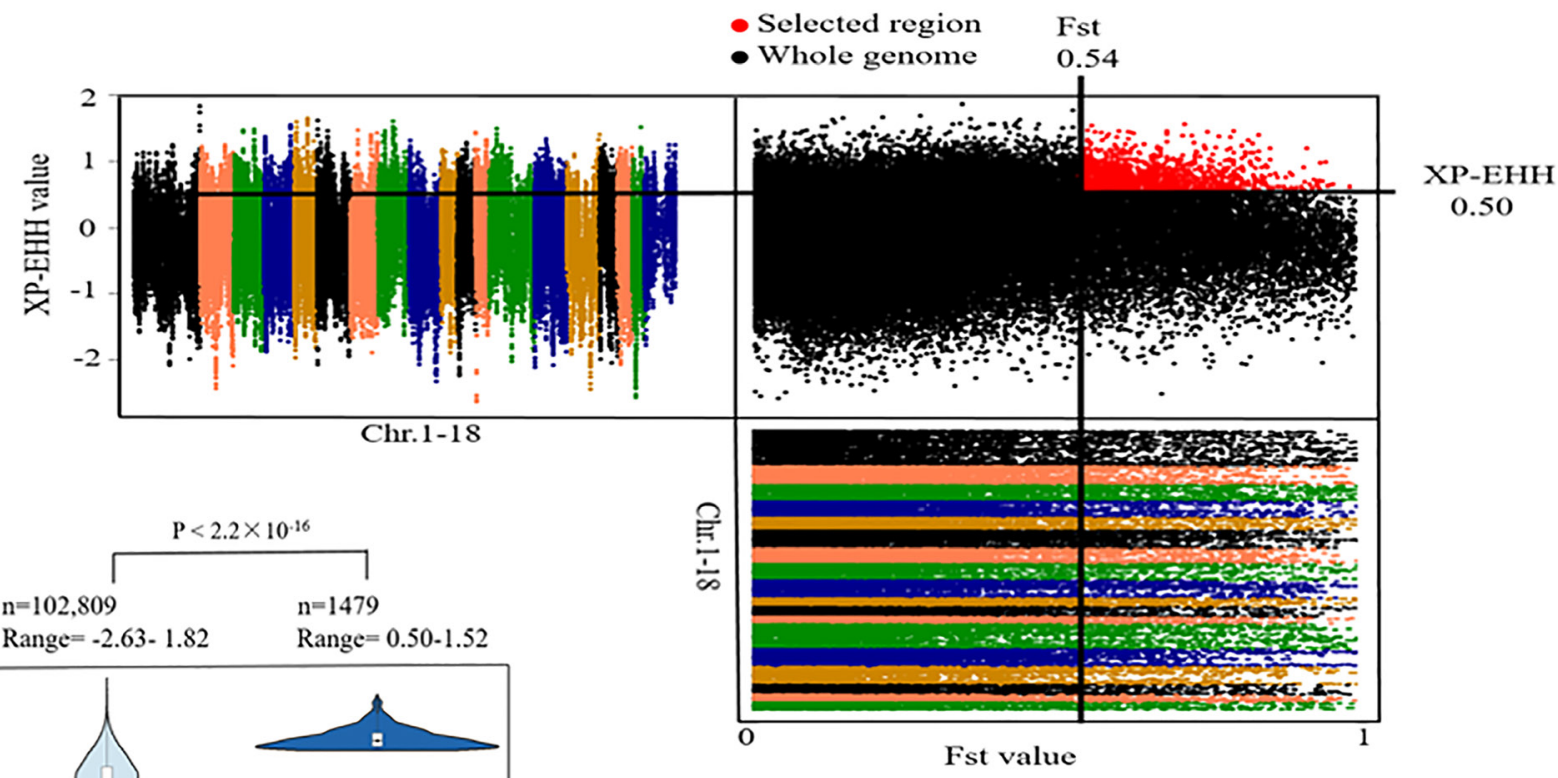

b

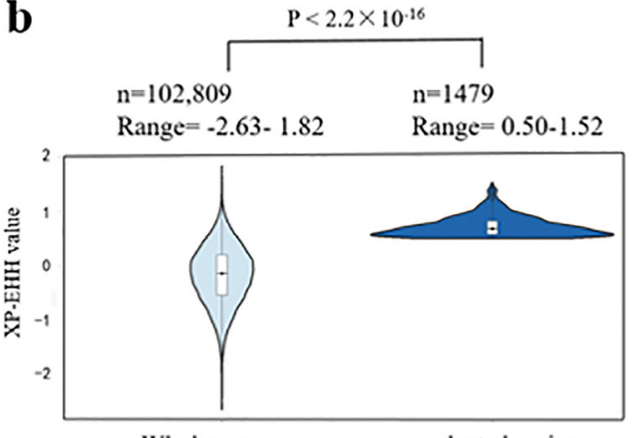

Fst value

Whole genome

selected regions

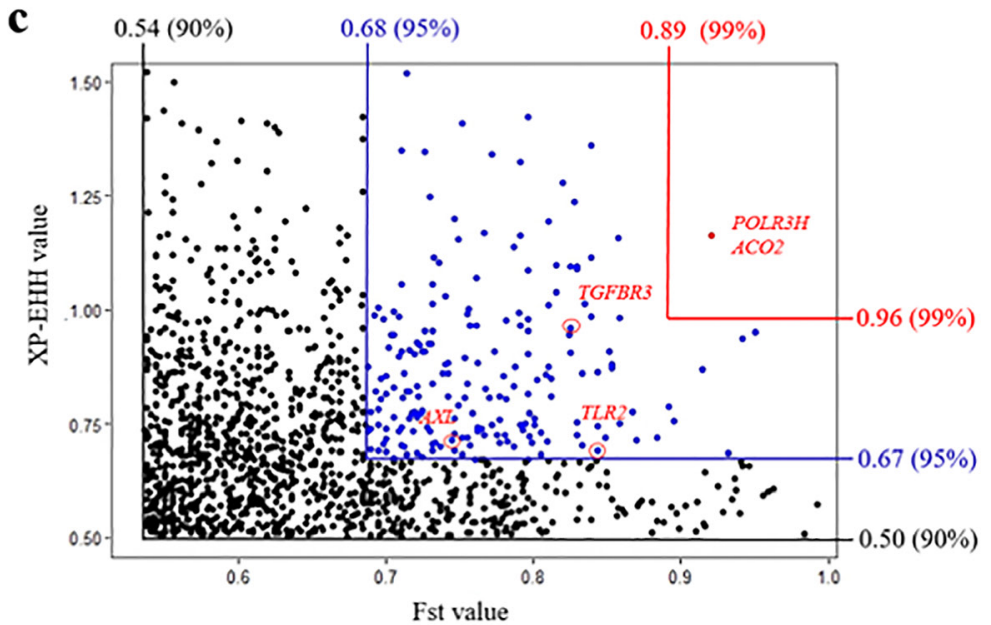

Figure 3. Genome-wide selective sweep analysis of group 1 (sensitive to swine enzootic pneumonia). (a) Distribution of XP-EHH and $F_{S T}$. Data points located to the right of the vertical lines ( $10 \%$ right tails of the $F_{S T}$ distribution, where $F_{S T}$ is 0.54$)$ and above the horizontal line ( $10 \%$ right tail of the XP-EHH distribution, where XP-EHH is 0.50$)$ were identified as selected regions for group 1 pigs (red points); (b) Violin plot of XP-EHH and $\mathrm{F}_{S \mathrm{ST}}$ values for regions of group 1 pigs that have undergone positive selection versus the whole genome regions. The statistical significance was calculated by the Mann-Whitney $\mathrm{U}$ test. (c) $10 \%, 5 \%$, and $1 \%$ significance level of XP-EHH and $\mathrm{F}_{\mathrm{ST}}$. XPEHH, cross-population extended haplotype homozygosity; ${ }_{F_{S T}}$ F-statistic.

EP-susceptibility mechanisms of Chinese indigenous pigs. Among the 691 genes with strong signatures of selection, there are 50 genes located in seven QTLs of three traits (Mycoplasmal pneumonia susceptibility, Mhp antibody titer and Change in Mhp antibody titer) (Supplementary Table S2), and 33 genes matched the GO terms associated with lung, immune, inflammatory response and drug metabolism (Supplementary Table S3), and then we merged genes of these two groups together as preliminary candidate genes. After literature search based on GeneCard and their biological functions, a set of 14 genes that have convincible biological functions associated with swine EP or human asthma come into the priority candidate genes (Table 2 and Supplementary Table S4). Among these
14 genes, previous researches have reported cytochrome $\mathrm{P} 450$ 1A1 (CYP1A1) and toll-like receptor 2 (TLR2) play an important role in the immune response to $M h p$ infection $[18,19]$.

Innate immune response and inflammatory response play an important role in pathogenicity of swine EP. AXL receptor tyrosine kinase $(A X L)$ regulates inhibition of toll-like receptors (TLRs)-mediated innate immune response and the DNA methylation of $A X L$ at birth was associated with higher risk for asthma-related symptoms in early childhood [20]. C-C motif chemokine ligand 11 (CCL11), an inflammatory cytokine, was significantly related to increased risk of asthma in adults [21]. Interleukin 7 receptor (IL7R) may control the adaptive immune response to PRRSV vaccine in Pietrain pigs 
Table 1. GO terms and KEGG pathways enriched with candidate genes

\begin{tabular}{llcc}
\hline Category & Term & Gene number & p-value (Benjamini) \\
\hline G0:0000267 & Cell fraction & 65 & 0.0022 \\
G0:0044425 & Membrane part & 274 & 0.0127 \\
G0:0016020 & Membrane & 296 & 0.0194 \\
KEGG:hsa00830 & Retinol metabolism & 13 & 0.0001 \\
KEGG:hsa00040 & Pentose and glucuronate interconversions & 7 & 0.0023 \\
KEGG:hsa00053 & Ascorbate and aldarate metabolism & 7 & 0.0023 \\
KEGG:hsa00150 & Androgen and estrogen metabolism & 8 & 0.0155 \\
KEGG:hsa00980 & Metabolism of xenobiotics by cytochrome P450 & 10 & 0.0190 \\
KEGG:hsa00983 & Drug metabolism & 8 & 0.0282 \\
KEGG:hsa00500 & Starch and sucrose metabolism & 8 & 0.0284 \\
KEGG:hsa00860 & Porphyrin and chlorophyll metabolism & 7 & 0.0304 \\
KEGG:hsa00140 & Steroid hormone biosynthesis & 8 & 0.0329 \\
KEGG:hsa00982 & Drug metabolism & 9 & 0.0419 \\
\hline
\end{tabular}

GO, gene ontology; KEGG, Kyoto encyclopedia of genes and genomes.

Table 2. Transcription analysis based on gene expression omnibus

\begin{tabular}{|c|c|c|c|c|c|c|c|c|}
\hline \multirow{2}{*}{ Candidate genes } & \multirow{2}{*}{ Chr } & \multirow{2}{*}{$\begin{array}{l}\text { Physical } \\
\text { position }\end{array}$} & \multicolumn{2}{|c|}{ Group1') } & \multicolumn{2}{|c|}{ Group2 ${ }^{1)}$} & \multicolumn{2}{|c|}{ Microarray } \\
\hline & & & AF1 & AF2 & AF1 & AF2 & $p$-value & $\log _{2}(F C)$ \\
\hline TCF7 & 2 & 142047175 & 0 & 1 & 0.488 & 0.512 & NF & NF \\
\hline EPAS1 & 3 & 100164175 & 0.024 & 0.976 & 0.694 & 0.306 & $3.38 \mathrm{E}-03$ & 1.32 \\
\hline TGFBR3 & 4 & 136718160 & 0.018 & 0.982 & 0.806 & 0.194 & NF & NF \\
\hline MMP2 & 6 & 27552563 & 0.003 & 0.997 & 0.5 & 0.5 & NF & $\mathrm{NF}$ \\
\hline$A X L$ & 6 & 44979578 & 0.324 & 0.676 & 0.997 & 0.003 & 7.32E-01 & 0.08 \\
\hline SPDEF & 7 & 35242328 & 0.128 & 0.872 & 0.8 & 0.2 & NF & NF \\
\hline CYP1A1 & 7 & 63474840 & 0.003 & 0.997 & 0.741 & 0.259 & NF & NF \\
\hline CYP1A2 & 7 & 63474840 & 0.003 & 0.997 & 0.741 & 0.259 & NF & NF \\
\hline CXCL2 & 8 & 74284832 & 0 & 1 & 0.529 & 0.471 & 3.10E-06 & 3.32 \\
\hline$T L R 2$ & 8 & 79818077 & 0.04 & 0.96 & 0.871 & 0.129 & $2.53 \mathrm{E}-03$ & 1.15 \\
\hline CCL11 & 12 & 42446570 & 0.476 & 0.524 & 0 & 1 & NF & NF \\
\hline CTNNB1 & 13 & 27684730 & 0 & 1 & 0.512 & 0.488 & $3.97 \mathrm{E}-01$ & 0.24 \\
\hline IL7R & 16 & 22326963 & 0.318 & 0.682 & 1 & 0 & $1.36 \mathrm{E}-04$ & 3.08 \\
\hline WNT2 & 18 & 30813337 & 0.11 & 0.89 & 0.771 & 0.229 & NF & $N F$ \\
\hline
\end{tabular}

AF, allele frequency; $F C$, fold change; NF, not found; TCF7, transcription factor 7; EPAS1, endothelial PAS domain protein 1; TGFBR3, transforming growth factor beta receptor 3; MMP2, matrix metallopeptidase 2; AXL, AXL receptor tyrosine kinase; SPDEF, SAM pointed domain containing ETS transcription factor; CYP1A1, cytochrome P450 1A1; CXCL2, C-X-C motif chemokine ligand 2; TLR2, toll-like receptor 2; CCL11, C-C motif chemokine ligand 11; CTNNB1, catenin beta 1; IL7R, interleukin 7 receptor; WNT2, Wnt family member 2.

1) Group1, Jinhua, Erhualian and Meishan pigs; Group2, Duroc and Landrace pigs.

by transcriptome data. Kurz et al [22] identified five asthma susceptibility loci including IL7R in German and Hutterite populations.

The abnormality of $\mathrm{Wnt} / \beta$-catenin signaling was believed to be associated with the development and pathogenesis of lung diseases. Wnt family member $2(W N T 2)$ and $\beta$-catenin 1 (CTNNB1) play a crucial role in the $\mathrm{Wnt} / \beta$-catenin signaling pathway $[23,24]$. Transcription factor 7 (TCF7) is one of important transcription factors for $\mathrm{T}$ cell development and differentiation, tumorigenesis, or embryonic development [25]. It is suggested to be involved in immune responses to lung diseases such as pulmonary infection, asthma, acute lung injury, emphysema and lung cancer through several signal pathways, especially the canonical Wnt $/ \beta$-catenin path- way [25].

The histochemistry and morphology of porcine airway cells would change in response to infection with swine EP. SAM pointed domain containing ETS transcription factor $(S P D E F)$ selectively expressed in respiratory epithelia cells is an important transcriptional factor of airway goblet cell differentiation and pulmonary inflammation in response to aeroallergens, and it can regulate a transcriptional network mediating mucus secretion in chronic airway disease [26]. Matrix metallopeptidase 2 (MMP2), involved in the inflammatory response, can mediate IL-13-induced suppression of elastin expression in airway fibroblasts [27]. CXCL2 and transforming growth factor beta receptor 3 (TGFBR3) play a role in the pathogenesis of airway remodeling in asthma [28]. 
Hypoxia is a fundamental component of respiratory disease, and the pressure of pulmonary and hematologic $\mathrm{O}_{2}$ and $\mathrm{CO}_{2}$ were decreased because of less oxygen consumption in swine with Mhp pneumonia [29]. Endothelial PAS domain protein 1 (EPAS1), also known as hypoxia inducible factor 2 subunit alpha, is a transcription factor that responds to hypoxia-responsive under high-altitude conditions in Tibetan pigs. Interestingly, a previous study reported that physiological hypoxia arises from lung alveoli suffering progressive airflow limitation which increases with pulmonary disease, chronic obstructive, severe early-onset (COPD) severity and EPAS1 is a key regulator of COPD through responding to hypoxia induced by airflow limitation as proved by integrative analysis of DNA methylation and gene expression data [30]. CYP1A2 is predominantly expressed in the liver which has been shown to modulate pulmonary oxygen toxicity. It plays a critical role in the susceptibility to hyperoxic lung injury by decreasing oxidative stress and lipid peroxidation in mice [31].

\section{Transcription analysis based on gene expression omnibus} To complement and verify the candidate genes we selected, the allele frequencies of loci in these candidate genes between two groups are shown in Table 2. And the only one published microarray of the porcine alveolar macrophage infected by swine EP was collected from GEO database, and the candidate genes were analysed. The p-value and $\log _{2}$ (FC) were listed in Table 2. Among 14 candidate genes, only six genes were found in the expression profiling array (Platforms GPL17577). Four of the six genes, EPAS1, CXCL2, TLR2, and IL7R, were significantly up-regulated (Figure 4 ).

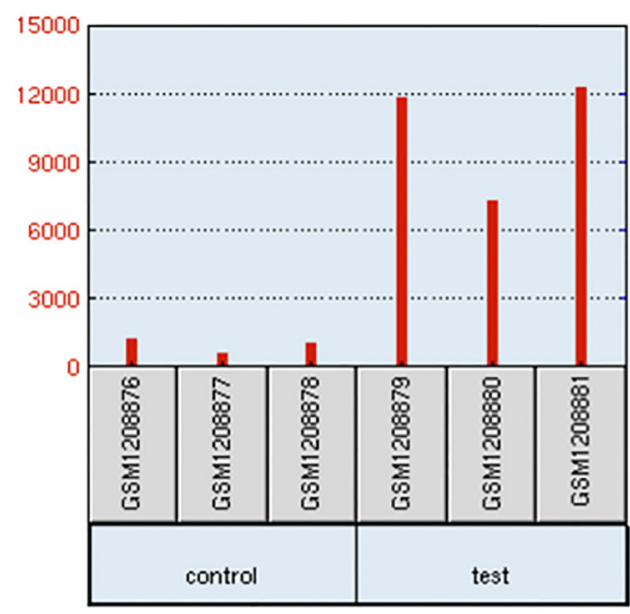

$\mathbf{a}$

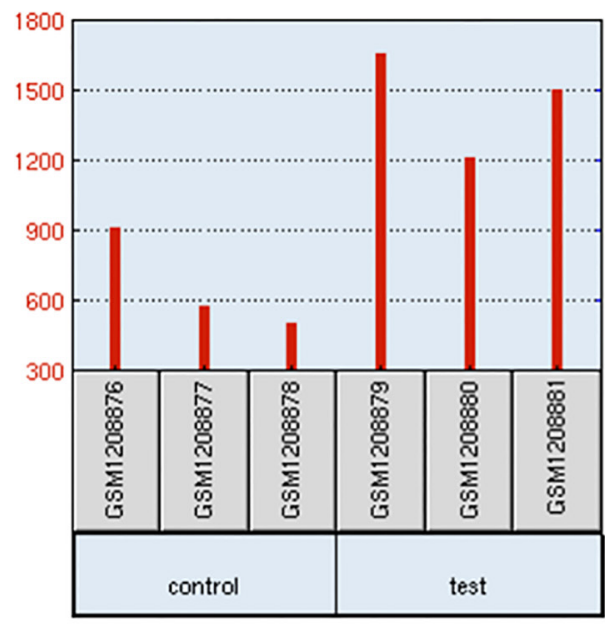

C
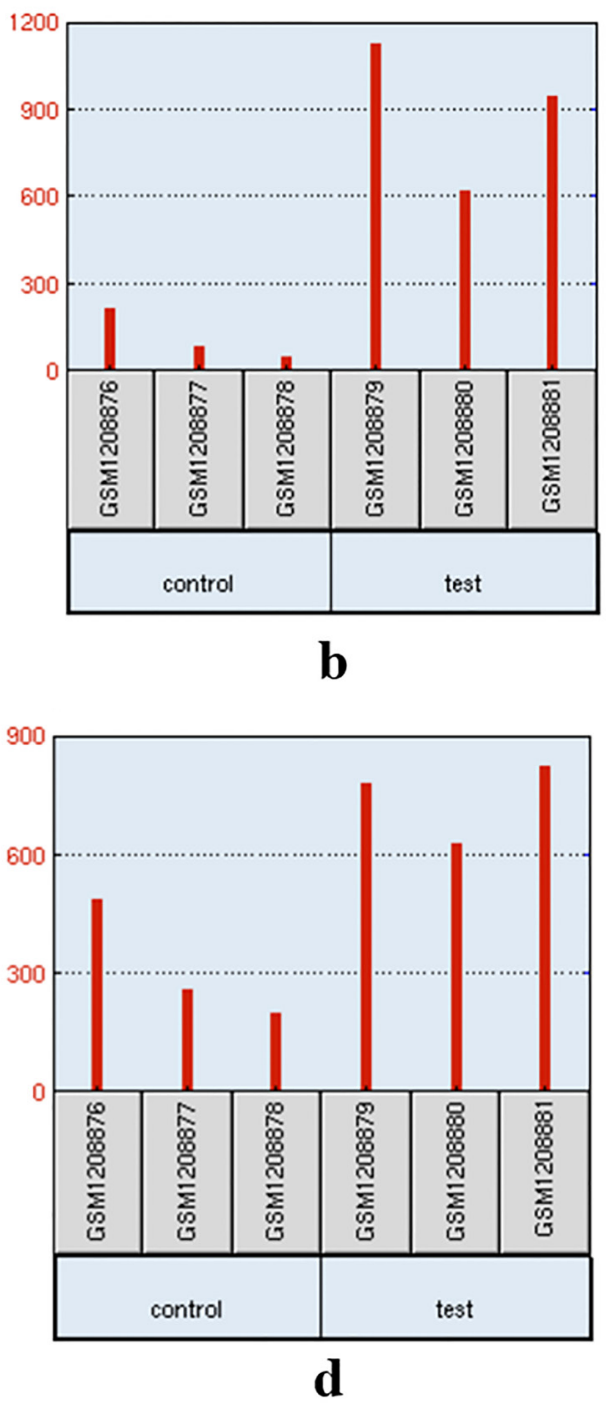

Figure 4. Expression of candidate genes in expression profiling array. (A) $C X C L 2$, (B) IL7R, (C) TLR2, and (D) EPAS1. Transcription analysis of the porcine alveolar macrophage response to infection of $M h p$ between test (6hMhp) and control (6hControl) cells. CXCL2, C-X-C motif chemokine ligand 2; IL7R, interleukin 7 receptor; TLR2, toll-like receptor 2; EPAS1, endothelial PAS domain protein 1; Mhp, Mycoplasma hyopneumoniae. 
Allele frequencies in candidate genes

After quantity control, we got 125,233 SNPs. Of the 171 Jinhua pigs, 93 pigs were grouped into controls (EP score $=0$ ) and 78 pigs were grouped into cases (EP score $\geq 1$ ). The allele frequencies in the 14 candidate genes were calculated between case and control groups. We found that the allele frequencies in six genes (TCF7, TGFBR3, SPDEF, CCL11, $I L 7 R$, and WNT2) were significantly different between those two groups (Supplementary Table S5). These results demonstrate the reliability of our results from another side.

The pleiotropic genes may be related to swine enzootic pneumonia susceptibility

Among above genes, some have other biological function involved in fertility, meat quality traits, or adipogenesis and fat deposition simultaneously. CYP1A1 can alter the activity of estrogen in the porcine ovary through estrogen receptor pathway [32]. CYP1A1 plays an important role in the human placenta during pregnancy. CTNNB1 as a molecular transcription factor is very important for the remodeling of uterine mucosa during development, the estrous cycle and early pregnancy in pigs [33]. CTNNB1 contributes to intercellular adhesion in large antral follicles and might influence the normal follicle development and pig fertility [34]. TGFBR3 plays a role in the muscular and adipose tissue development and it is a candidate gene involved in meat quality traits in pigs [8].

WNT2, CTNNB1, and TCF7 are all involved in the Wnt/ $\beta$-catenin signaling pathway, and this pathway plays a critical role in regulating porcine adipogenesis and fat deposition. Chinese indigenous breeds display a higher intramuscular fat content and more abdominal fat deposition than commercial Western pig breeds. And thus, these genes involved in fat deposition may be selected during the natural and artificial selection while they are simultaneously involved in susceptibility to EP disease in pigs. In a previous study, Huang et al also suggested that genes involved in fat deposition may play an important role in susceptibility or resistance to the EP disease in pigs [4]. Additionally, many epidemiologic studies have found that abdominal obesity increases the risk of developing asthma in humans [35].

\section{Linked genes with candidate genes may be related to} swine enzootic pneumonia susceptibility

The LD scores between SNPs located in these candidate genes and the other SNPs in selected regions were calculated. Three genes subjected to strong $\mathrm{LD}\left(\mathrm{r}^{2} \geq 0.8\right)$ are listed in Table 3 . Specifically, seven and two loci on chromosomes 6 were completely linked to $A X L$ and $M M P 2$ respectively. In addition, two loci on chromosomes 7 were strongly linked to SPDEF (Table 3).

Plasminogen activator, urokinase receptor (PLAUR) (completely linked to $A X L$ ) also known as $C D 87$, is a part of the plasminogen activation system, which regulates the conversion of plasminogen to plasmin. PLAUR plays an essential role in the successful ovulation of matured oocytes together with follicle stimulating hormone in pigs [36]. Colipase like 2 (CLPSL2) (strongly linked to SPDEF, $\mathrm{r}^{2}=0.848$ ), specifically expressed in epididymis, was involved in the regulation of acrosomal integrity, spermatozoa motility, and male fertility. Knockdown of CLPSL2 expression in mouse epididymis results in a decreased number of sperm with intact acrosome, attenuated sperm motility, and reduced the fertility [37].

We noticed that these Chinese indigenous pig breeds show an excellent fertility and meat quality, higher intramuscular fat content and more abdominal fat deposition compared to

Table 3. Strong LD between SNPs located in candidate genes and the other SNPs in selected regions

\begin{tabular}{|c|c|c|c|c|c|c|}
\hline Chr & Physical position & Genes & Chr & Physical position & $\operatorname{LD}\left(r^{2}\right)$ & Associated genes \\
\hline \multirow[t]{2}{*}{6} & 27552563 & MMP2 & 6 & 27451454 & 1 & LPCAT2, SLC6A2 \\
\hline & & & 6 & 27451485 & 1 & LPCAT2, SLC6A2 \\
\hline \multirow[t]{7}{*}{6} & 44979578 & $A X L$ & 6 & 45100366 & 1 & CCDC97, HNRNPUL1 \\
\hline & & & 6 & 45487407 & 1 & MEGF8 \\
\hline & & & 6 & 45601909 & 1 & GSK3A, CYP2B6 \\
\hline & & & 6 & 46185803 & 1 & IRGQ, XRCC1, ZNF576 \\
\hline & & & 6 & 46185804 & 1 & $I R G Q, X R C C 1, Z N F 576$ \\
\hline & & & 6 & 46185819 & 1 & $I R G Q, X R C C 1, Z N F 576$ \\
\hline & & & 6 & 46331807 & 1 & PLAUR, CADM4 \\
\hline \multirow[t]{2}{*}{7} & 35242328 & SPDEF & 7 & 35722329 & 0.881 & ANKS1A \\
\hline & & & 7 & 36516210 & 0.848 & CLPSL2, ARMC12 \\
\hline
\end{tabular}

LD, linkage disequilibrium; SNPs, single nucleotide polymorphisms; MMP2, matrix metallopeptidase 2; LPCAT2, lysophosphatidylcholine acyltransferase 2; SLC6A2, solute carrier family 6 member 2; AXL, AXL receptor tyrosine kinase; CCDC97, coiled-coil domain containing 97; HNRNPUL1, heterogeneous nuclear ribonucleoprotein U like 1; MEGF8, multiple EGF like domains 8; GSK3A, glycogen synthase kinase 3 alpha; CYP2B6, cytochrome P450 family 2 subfamily B member 6; IRGQ, immunity related GTPase Q; XRCC1, $X$-ray repair cross complementing 1; ZNF576, zinc finger protein 576; PLAUR, plasminogen activator, urokinase receptor; CADM4, cell adhesion molecule 4; SPDEF, SAM pointed domain containing ETS transcription factor; ANKS1A, ankyrin repeat and sterile alpha motif domain containing 1A; CLPSL2, colipase like 2; ARMC12, armadillo repeat containing 12. 
European breeds under the natural and artificial selective pressure. These adverse genes of susceptibility to swine EP may be selected simultaneously while selecting economic traits due to pleiotropic effects and hitchhiking effect of linked genes. These results support our hypothesis to a great extent and provide a completely new point of view to explain why Chinese indigenous pig breeds are more sensitive to swine EP.

In conclusion, this study revealed several candidate genes that are involved in the susceptibility to swine EP including TCF7, EPAS1, TGFBR3, MMP2, AXL, SPDEF, CYP1A1, CYP1A2, CXCL2, TLR2, CCL11, CTNNB1, IL7R, and WNT2 from positive selection signature. Most of these genes are involved in inflammatory response, hypoxia-responsive or $\mathrm{Wnt} / \beta$-catenin signaling pathway. The susceptibility loci affecting swine EP have increased during the selection for fertility and meat quality traits because of pleiotropic and hitch-hiking effects. These findings will help in increasing our understanding of the genetic basis for susceptibility or resistance to EP and other respiratory diseases in pigs thereby, provide insight for designing sustainable breed selection programs (such as marker assisted selection). Moreover, we provided a completely new point of view to explain why Chinese indigenous pig breeds are more sensitive to swine EP. In addition, exploring the molecular mechanisms of the susceptibility to swine EP may be crucial for its potential role in human asthma and other respiratory diseases.

\section{AUTHOR CONTRIBUTIONS}

Pan YC and Wang QS designed and supervised the study, while $\mathrm{Xu} \mathrm{Z}$ analyzed the data with the help of Sun $\mathrm{H}$, Zhang $\mathrm{Z}$, Zhao QB and Zhang CY, Xu Z wrote the article with the help of Xiao Q, Olasege BS, Ma PP, and Zhang XZ. All authors read and edited the article.

\section{CONFLICT OF INTEREST}

We certify that there is no conflict of interest with any financial organization regarding the material discussed in the manuscript.

\section{ACKNOWLEDGMENTS}

This work was supported by Zhejiang province agriculture (livestock) varieties breeding Key Technology R\&D Program (Grant No. 2016C02054-2), National Natural Science Foundation of China (No. 31872976, U1402266).

\section{REFERENCES}

1. Simionatto S, Marchioro SB, Maes D, Dellagostin OA. Myco- plasma hyopneumoniae: from disease to vaccine development. Vet Microbiol 2013;165:234-42. https://doi.org/10.1016/j. vetmic.2013.04.019

2. Hong SJ. The role of Mycoplasma pneumoniae infection in asthma. Allergy Asthma Immunol Res 2012;4:59-61. http:// dx.doi.org/10.4168/aair.2012.4.2.59

3. Okamura T, Onodera W, Tayama T, et al. A genome-wide scan for quantitative trait loci affecting respiratory disease and immune capacity in Landrace pigs. Anim Genet 2012;43:7219. https://doi.org/10.1111/j.1365-2052.2012.02359.x

4. Huang X, Huang T, Deng W, et al. Genome-wide association studies identify susceptibility loci affecting respiratory disease in Chinese Erhualian pigs under natural conditions. Anim Genet 2017;48:30-7. https://doi.org/10.1111/age.12504

5. Fang X, Zhao W, Fu Y, et al. Difference in susceptibility to mycoplasma pneumonia among various pig breeds and its molecular genetic basis. Sci Agric Sin 2015;48:2839-47. https:// doi.org/10.3864/j.issn.0578-1752.2015.14.015

6. Sabeti PC, Varilly P, Fry B, et al. Genome-wide detection and characterization of positive selection in human populations. Nature 2007;449:913-8. https://doi.org/10.1038/nature06250

7. Weir BS, Cockerham CC. Estimating F-statistics for the analysis of population structure. Evolution 1984;38:1358-70. https:// doi.org/10.1111/j.1558-5646.1984.tb05657.x

8. Jeong $\mathrm{H}$, Song KD, Seo M, et al. Exploring evidence of positive selection reveals genetic basis of meat quality traits in Berkshire pigs through whole genome sequencing. BMC Genet 2015;16: 104. https://doi.org/10.1186/s12863-015-0265-1

9. Taye M, Lee W, Caetano-Anolles K, et al. Whole genome detection of signature of positive selection in African cattle reveals selection for thermotolerance. Anim Sci J 2017;88: 1889-901. https://doi.org/10.1111/asj.12851

10. Yuan Z, Liu E, Liu Z, et al. Selection signature analysis reveals genes associated with tail type in Chinese indigenous sheep. Anim Genet 2017;48:55-66. https://doi.org/10.1111/age.12477

11.Li Z, Chen J, Wang Z, et al. Detection of selection signatures of population-specific genomic regions selected during domestication process in Jinhua pigs. Anim Genet 2016;47:672-81. https://doi.org/10.1111/age.12475

12. Wang Z, Chen Q, Yang Y, et al. Genetic diversity and population structure of six Chinese indigenous pig breeds in the Taihu Lake region revealed by sequencing data. Anim Genet 2015;46:697-701. https://doi.org/10.1111/age.12349

13.Zhang Z, Wang Z, Yang Y, et al. Identification of pleiotropic genes and gene sets underlying growth and immunity traits: a case study on Meishan pigs. Animal 2016;10:550-7. https:// doi.org/10.1017/S1751731115002761

14.Chen Q, Ma Y, Yang Y, et al. Genotyping by genome reducing and sequencing for outbred animals. PloS One 2013;8:e67500. https://doi.org/10.1371/journal.pone.0067500

15.Purcell S, Neale B, Todd-Brown K, et al. PLINK: a tool set for whole-genome association and population-based linkage 
analyses. Am J Hum Genet 2007;81:559-75. https://doi.org/ $10.1086 / 519795$

16.Li B, Du L, Xu X, et al. Transcription analysis on response of porcine alveolar macrophages to co-infection of the highly pathogenic porcine reproductive and respiratory syndrome virus and Mycoplasma hyopneumoniae. Virus Res 2015;196:609. https://doi.org/10.1016/j.virusres.2014.11.006

17.Tsui KH, Chung LC, Wang SW, Feng TH, Chang PL, Juang $\mathrm{HH}$. Hypoxia upregulates the gene expression of mitochondrial aconitase in prostate carcinoma cells. J Mol Endocrinol 2013;51:131-41. https://doi.org/10.1530/JME-13-0090

18. Fang X, Liu X, Meng C, et al. Breed-linked polymorphisms of porcine toll-like receptor 2 (TLR2) and TLR4 and the primary investigation on their relationship with prevention against Mycoplasma pneumoniae and bacterial LPS challenge. Immunogenetics 2013;65:829-34. https://doi.org/10.1007/ s00251-013-0727-4

19. Fang X, Zhao W, Xu J, et al. CYP1A1 mediates the suppression of major inflammatory cytokines in pulmonary alveolar macrophage (PAM) cell lines caused by Mycoplasma hyponeumoniae. Dev Comp Immunol 2016;65:132-8. https://doi.org/10.1016/ j.dci.2016.06.023

20. Gao L, Millstein J, Siegmund KD, et al. Epigenetic regulation of $A X L$ and risk of childhood asthma symptoms. Clin Epigenetics 2017;9:121. https://doi.org/10.1186/s13148-017-0421-8

21.Zhou T, Huang X, Zhou Y, et al. Associations between Th17related inflammatory cytokines and asthma in adults: A CaseControl Study. Sci Rep 2017;7:15502. https://doi.org/10.1038/ s41598-017-15570-8

22. Kurz T, Hoffjan S, Hayes MG, et al. Fine mapping and positional candidate studies on chromosome 5 p13 identify multiple asthma susceptibility loci. J Allergy Clin Immunol 2006;118: 396-402. https://doi.org/10.1016/j.jaci.2006.04.036

23. Valenta T, Hausmann G, Basler K. The many faces and functions of beta-catenin. EMBO J 2012;31:2714-36. https://doi. org/10.1038/emboj.2012.150

24.Li N, Li S, Wang Y, et al. Decreased expression of WNT2 in villi of unexplained recurrent spontaneous abortion patients may cause trophoblast cell dysfunction via downregulated Wnt/beta-catenin signaling pathway. Cell Biol Int 2017;41:898907. https://doi.org/10.1002/cbin.10807

25.Zhu Y, Wang W, Wang X. Roles of transcriptional factor 7 in production of inflammatory factors for lung diseases. J Transl Med 2015;13:273. https://doi.org/10.1186/s12967-015-0617-7

26.Liu Z, Chen X, Wu Q, Song J, Wang L, Li G. miR-125b inhibits goblet cell differentiation in allergic airway inflammation by targeting SPDEF. Eur J Pharmacol 2016;782:14-20. https:// doi.org/10.1016/j.ejphar.2016.04.044
27.Ingram JL, Slade D, Church TD, et al. Role of matrix metalloproteinases-1 and -2 in interleukin-13-suppressed elastin in airway fibroblasts in asthma. Am J Respir Cell Mol Biol 2016; 54:41-50. https://doi.org/10.1165/rcmb.2014-0290OC

28. Al-Alwan LA, Chang Y, Mogas A, et al. Differential roles of CXCL2 and CXCL3 and their receptors in regulating normal and asthmatic airway smooth muscle cell migration. J Immunol 2013;191:2731-41. https://doi.org/10.4049/jimmunol.1203421

29. Intraraksa Y, Engen RL, Switzer WP. Pulmonary and hematologic changes in swine with Mycoplasma hyopneumoniae pneumonia. Am J Vet Res 1984;45:474-7.

30. Yoo S, Takikawa S, Geraghty P, et al. Integrative analysis of DNA methylation and gene expression data identifies EPAS1 as a key regulator of COPD. PLoS Genet 2015;11:e1004898. https://doi.org/10.1371/journal.pgen.1004898

31. Wang L, Lingappan K, Jiang W, et al. Disruption of cytochrome P4501A2 in mice leads to increased susceptibility to hyperoxic lung injury. Free Radic Biol Med 2015;82:147-59. https://doi. org/10.1016/j.freeradbiomed.2015.01.019

32.Ptak A, Ludewig G, Gregoraszczuk EL. A low halogenated biphenyl (PCB3) increases CYP1A1 expression and activity via the estrogen receptor beta in the porcine ovary. J Physiol Pharmacol 2008;59:577-88.

33. Kiewisz J, Kaczmarek MM, Andronowska A, Blitek A, Ziecik AJ. Gene expression of WNTs, beta-catenin and E-cadherin during the periimplantation period of pregnancy in pigs--involvement of steroid hormones. Theriogenology 2011;76:68799. https://doi.org/10.1016/j.theriogenology.2011.03.022

34. Grzesiak M, Knapczyk-Stwora K, Duda M, Slomczynska M. Elevated level of 17beta-estradiol is associated with overexpression of FSHR, CYP19A1, and CTNNB1 genes in porcine ovarian follicles after prenatal and neonatal flutamide exposure. Theriogenology 2012;78:2050-60. https://doi.org/10.1016/ j.theriogenology.2012.07.026

35. Diaz J, Warren L, Helfner L, et al. Obesity shifts house dust mite-induced airway cellular infiltration from eosinophils to macrophages: effects of glucocorticoid treatment. Immunol Res 2015;63:197-208. https://doi.org/10.1007/s12026-015$8717-2$

36. Blaha M, Nemcova L, Kepkova KV, Vodicka P, Prochazka R. Gene expression analysis of pig cumulus-oocyte complexes stimulated in vitro with follicle stimulating hormone or epidermal growth factor-like peptides. Reprod Biol Endocrinol 2015; 13:113. https://doi.org/10.1186/s12958-015-0112-2

37.Lu X, Ding F, Lian Z, et al. An epididymis-specific secretory protein Clpsl2 critically regulates sperm motility, acrosomal integrity, and male fertility. J Cell Biochem 2018;119:4760-74. https://doi.org/10.1002/jcb.26668 